\title{
Advanced OoA and Automated Technologies for the Manufacturing of a Composite Outer Wing Box
}

\author{
Fulvio Romano 1,*, Marco Barile ${ }^{2}$, Gianpaolo Cacciapuoti ${ }^{3}$,Jean-Luc Godard ${ }^{4}$, Paolo \\ Vollaro $^{5}$, and Philippe Barabinot ${ }^{6}$ \\ ${ }^{1}$ CIRA - Italian Aerospace Research Centre, Via Maiorise snc, 81043 Capua (CE), Italy \\ ${ }^{2}$ NOVOTECH Aerospace Advanced Technology S.r.l., 80125 Napoli, Italy \\ ${ }^{3}$ SICAMB, Strada Eschido 1, 04100 Latina, Italy \\ ${ }^{4}$ ONERA - The French Aerospace Lab, F-92190 Meudon, France \\ ${ }^{5}$ IMAST - Polymeric and Composite Materials and Structures, P.zza Bovio 22, 80133 Napoli, Italy \\ ${ }^{6}$ Siemens Industry Software SAS, 150 Avenue de la République, 92320 Chatillon, France
}

\begin{abstract}
This work resumes the results achieved until today in the European project AirGreen 2 of Clean Sky 2 programme, deriving from the application of two different dry preforming processes for the manufacturing of a composite outer wing box of the next generation turboprop aircrafts. Liquid Resin Infusion and Out of Autoclave techniques, by Hand-Layup and Automated Fiber Placement, are considered. The optimisation and validation of the manufacturing processes have been done according to key performance indexes: weight and cost reduction, lower energy consumption, high productivity and minimal reworking time, less intensive labour, minimal scrap and less waste of materials. The work has been performed through manufacturing tests and optimisation of the process parameters, implementation of several bagging techniques, numerical simulations of the infusion process and material characterization tests in operative conditions, from coupons level up to details and elements level (flat stiffened panels). Pro and cons, suggestions and technical considerations useful for the next step of the project (final manufacturing of large parts and components) are assessed.
\end{abstract}

\section{Introduction}

The AirGreen 2 project is an Innovation Action funded by the Clean Sky 2 Joint Undertaking under the European Union's Horizon 2020 research and innovation programme, under Grant Agreement n. CS2-REG-GAM-2014-2015-01. One of the main scopes of the project is the "econolomic" manufacturing of the Outer Wing Box (OWB) of a Regional Turboprop Aircraft. The Liquid Resin Infusion (LRI) and Out of Autoclave (OoA) manufacturing process is the focus of the activity, starting from some validations already reached at laboratory level in the previous project AirGreen of Clean Sky (CS1), [1]. Hand-

\footnotetext{
* Corresponding author: f.romano@,cira.it
} 
Layup (HLU) and Automated Fiber Placement (AFP) dry preforming processes are applied, by improving weight and cost reduction, lower energy consumption, high productivity and minimal (manual) reworking time, less intensive labour, minimal scrap and lower buy/fly ratio. NOVOTECH has validated and verified the great potential of the liquid resin infusion process based on preforms laydown with the AFP technology [2]. SICAMB, involved in the HLU process, has also optimized the infusion process through a commercial software (ESIPAM/RTM, [3]) varying the models with different inlet/outlet configurations to verify the best solution to obtain the full infusion with the shortest process time. Besides, the vulnerability to thermal loads and to lightning has been tested by IMAST, ONERA, CIRA and SAMTECH experimentally and numerically. Technical considerations useful for the next manufacturing of large parts and components, are assessed.

\section{Manufacturing improvement and development}

The optimisation of the LRI/OoA process has passed through the production of several coupons, elements and mid-scale details, up to the manufacturing of the flat stiffened panel illustrated in Figure 1(a) for both the HLU and AFP techniques. This panel is representative of the upper skin at the wing tip, Figure 1(b). In particular the panel $(950 \times 480 \mathrm{~mm})$ has a skin of 14 plies [45/-45/0/0/90/-45/45] s, and 3 stringers with 10 plies spaced of $150 \mathrm{~mm}$ : same thickness for both the web and the flanges. The two material systems are characterised by the same resin, PRISM EP2400, while for the fiber: BNCF-24KIMS-(0)-196-600 for the HLU and TX1100 IMS65-24K-UD-196-6.35 for the AFP.

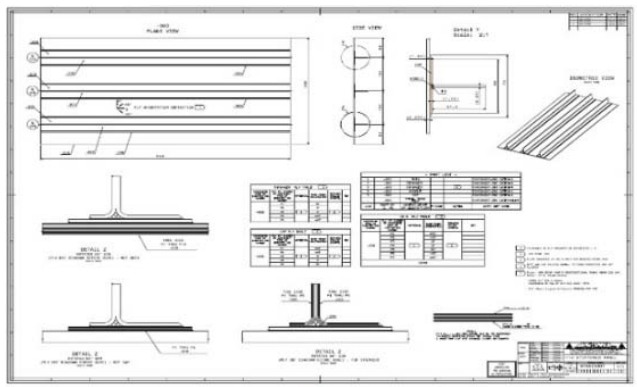

(a)

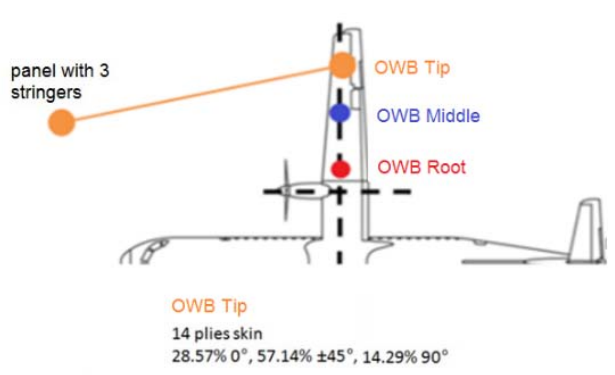

(b)

Fig. 1. Flat stiffened panel.

\subsection{LRI optimisation and HLU technique}

The LRI technique has been optimised by the development of dedicated mechanical/fluid dynamic models, simulating the liquid resin flow through stiffened wing panels preform and assisting equipment and tools design. PAM RTM software has been used for this scope; it integrates thermo-chemistry and viscosity models as appropriate. The activity is aimed at identifying possible causes of the slow down or non-uniform distribution of resin flow that may cause dry spots, poor saturation of the preform and partially filled composite parts.

The LRI model is based on Darcy's law that governs the flow of a liquid resin through porous media. PAM RTM has supported the evaluation of different impregnation strategies and the identification of optimum key process parameters: the location of distribution media, inlet and outlet pipes and impregnation temperature. The characteristic parameters are the viscosity and permeability, and the isothermal process is dictated by the process conditions on pressure, volume flow rate and vents. Figure 2(a) shows the FE model of the panel and Figure 2(b) the regions on which are applied the process conditions. Appropriate tests has 
been performed to different temperatures in order to obtain a good experimental-numerical correlation.
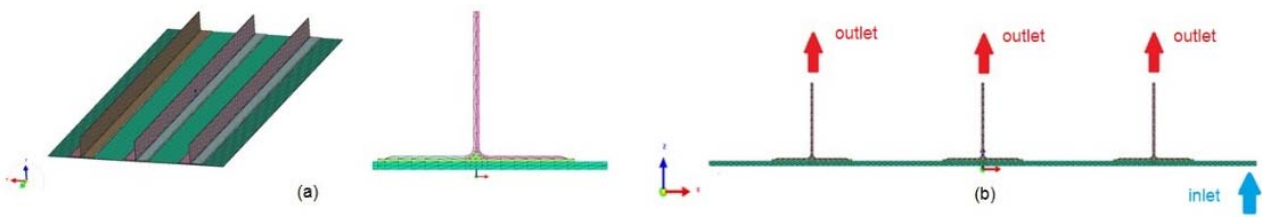

Fig. 2. Flat stiffened panel FE model and process condition regions.

The permeability characterisation tests, with $\triangle P=50 \mathrm{kPa}$, have shown for the HLU carbon preform an average permeability of $5.8 \cdot 10^{-11} \mathrm{~m}^{2}$, a standard deviation of $2.0 \cdot 10^{-13}$ and a deviation coefficient of only 0.36 . Dedicated tests has given for PRISM EP2400 resin approximated values of the gel time, initial viscosities, and the time to reach a viscosity $(\mu)$ of the resin system of $300 \mathrm{cP}$ (reference value for the permeation of the resin through a carbon fabric preform: $\left.100^{\circ} \mathrm{C}, \mu=0.1 \mathrm{~Pa} \cdot \mathrm{s}\right)$. The characteristics were collected at different temperatures (from $100^{\circ} \mathrm{C}$ up to $185^{\circ} \mathrm{C}$ ) and compared; the results are in accordance to the Arrhenius law.

Three numerical cases has been considered for the FE model of Figure 2(a). Several tests with different temperatures (i.e. different viscosities for the same FE model). The inlet pressure is $10^{6} \mathrm{~Pa}$, the outlet pressure is $0 \mathrm{~Pa}$, and the viscosity is respectively $0.2125 \mathrm{~Pa} \cdot \mathrm{s}$, $0.425 \mathrm{~Pa} \cdot \mathrm{s}, 0.85 \mathrm{~Pa} \cdot \mathrm{s}$. The experimental time is resulted $49 \mathrm{~min}$. The simulation time, with viscosity equal to 0.425 (case 2), is resulted $44 \mathrm{~min}$, very close to the experimental value. Figure 3(a) and 3(b) illustrate respectively the filling time and pressure trend for this case and Figure 3(c) the final optimised resin inlet/outlet scheme.
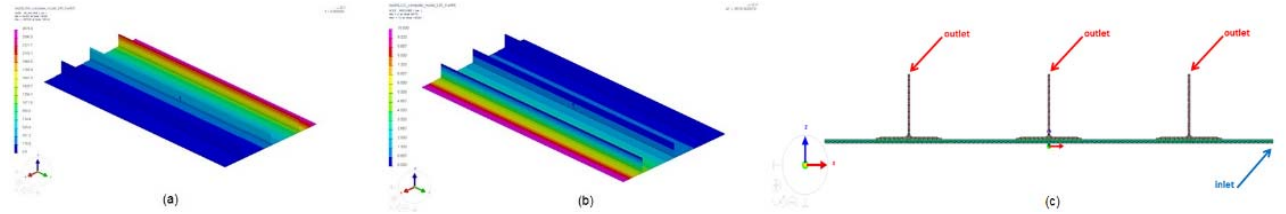

Fig. 3. Flat stiffened panel: FE model and process condition regions.

The HLU process procedure of SICAMB foresees: panel preparation, bagging, resin heating, resin degassing, infusion and cure. Figure 4(a) shows the precompaction scheme of the skin (without stringers): laminate laid up, bag preparation and leak test, compaction in oven (1 hour at $80-90^{\circ} \mathrm{C}$ ). The same procedure is applied for the stringers that then are assembled with the skin. Figure 4(b) shows the final optimised scheme of the vacuum bag. Figure 5(a) shows autoclave (without pressure) infusion process, Figures 5(b) and 5(c) the final panel with the $\mathrm{C}$-scan results.

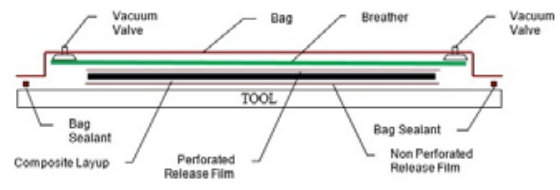

(a)

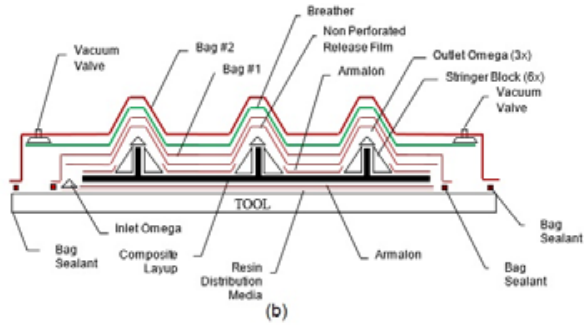

Fig. 4. Flat stiffened panel: precompacted scheme of the skin and vacuum bag scheme. 


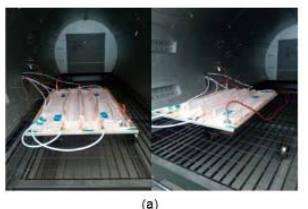

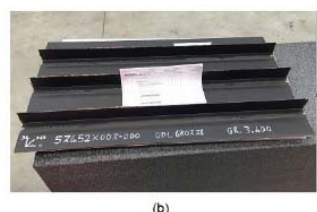

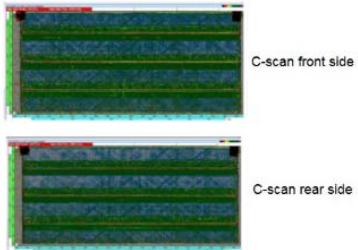

Fig. 5. HLU flat stiffened panel: autoclave (without pressure) infusion process, final panel, C-scan.

\subsection{AFP technique}

The production of the abovementioned composite stiffened panel has been conceived according to a bottom-up approach, starting from a small scale demo consisting of a monostringer panel, up to the production of the final full scale flat stiffened panel. Figure 6(a) and 6(b) illustrate the final AFP manufactured panel with C-scan imagines.

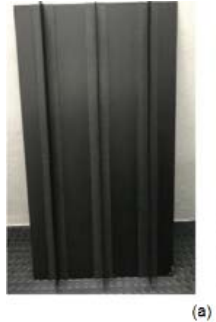

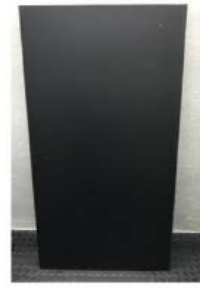

(a)

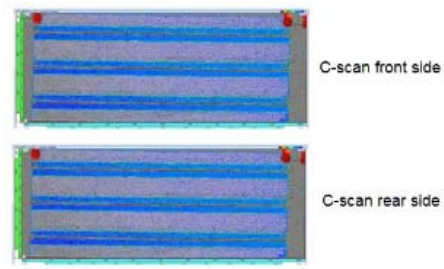

(b)

Fig. 6. AFP flat stiffened panel with C-scan imagines.

The manufacturing has been done by using a laser assisted fibre placement robot [4], developed by Coriolis Composites (France) and available at NOVOTECH's plant, Figure 7(a).

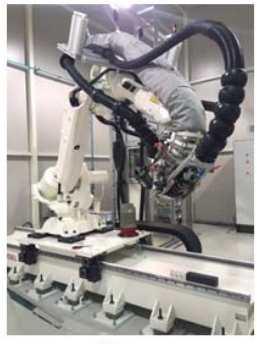

(a)
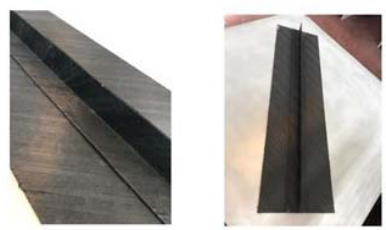

(b)
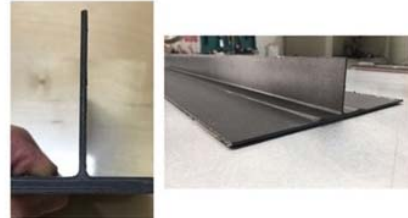

(c)

Fig. 7. Coriolis AFP machine, mono-stringer and AFP preform, mono-stringer demo infused.

Then, the dry preforms are infused by LRI process. The AFP cell is equipped with a $3 \mathrm{~kW}$ laser optics allowing an increased control of the temperature needed for the heating of the tapes. The improved optics, combined with an enhanced control of the main process parameters (lay-down speed and temperature, head compaction force, etc.) is able to increase the quality of the part produced. This machine is composed of an ABB IRB6640 standard 7axes robot, mounted on a 4.5 meter linear axis. The layup head used is an $8 \times 6.35 \mathrm{~mm}$ tow head (maximum bandwidth of $50.8 \mathrm{~mm}$ ). The compaction is performed by a suitable roller that guarantees a force of $500 \mathrm{~N}$. The contact surface of the roller is approximately $55 \mathrm{~mm} \times$ $15 \mathrm{~mm}$. Therefore the average contact pressure applied is around $0.6 \mathrm{MPa}$ and it is limited by the stiffness of the robot. The programming of the panels is performed using the Coriolis 
CADFiber software. The small scale demo has been useful to set the AFP preforming process and infusion strategies (injection lines, bagging scheme, etc.). With regard to the infusion process, a composite curing oven coupled with an advanced online monitoring system developed from BriskHeat Corporation for the control of the manufacturing process parameters (temperatures, vacuum level) has been used. Similar systems have been developed in the past [5]. Figure 7(b) and 7(c) show the dry preform of the mono-stringer demo and after infusion respectively.

\subsection{Main outcomes of the manufacturing activities}

The manufacturing and simulation activities performed in the project have given the possibility to evaluate and compare the two above techniques. In terms of material waste, the average waste of dry fabric is resulted of about $30 \%$ for the HLU and of no more than $5 \%$ for the AFP. Regarding the ply placement, the HLU layup speed is resulted much affected by the number of ply segments composing the layer and decreases with bigger size ply segments, while for the AFP the layup speed is constant, regardless the ply size (intermediate compactions are not required).The HLU vs AFP laydown rate ratio for non-serial parts is resulted of about 1:4. In terms of compaction, the HLU requires a separate final compaction during which the complete layup (including the tool) is warmed-up to about $120^{\circ} \mathrm{C}$ in oven and under vacuum bag, including the mould tool. The energy required for this process is mainly driven by the mass of the tool. AFP does not require the final compaction (if layup is done directly on the mold tool). The fibers are warmed-up and compacted during the placement. The heat is used to warm-up the fiber (binder) only. HLU process is not affected by the shape of the final composite part. AFP process is effective for planar or very large curvature radius surfaces (skins); it is not recommended for small curvature shapes. The amount of energy required for the LRI production phases (common for HLU and AFP) is resulted of $243.114 \mathrm{kWh}$ (sum of consumptions: transport, storage, processing).

\section{Vulnerability in operative conditions}

The vulnerability to critical thermal scenarios has been investigated in the project for the two selected material systems. Experimental tests according to ASTM regulations and numerical simulations have been performed. The thermal operative scenarios of the OWB have been identified: the thermal loads that could occur have been identified as well as the regions of the OWB subjected to these loads. Figure 8(a) shows the thermal critical scenarios, while Figure $8(\mathrm{~b})$ the aircraft zoning and currents for lightning. Three representative laminates of the OWB have been considered: 14,24 and 30 plies $(2.9 \mathrm{~mm}, 4.9 \mathrm{~mm}, 6.1 \mathrm{~mm})$.
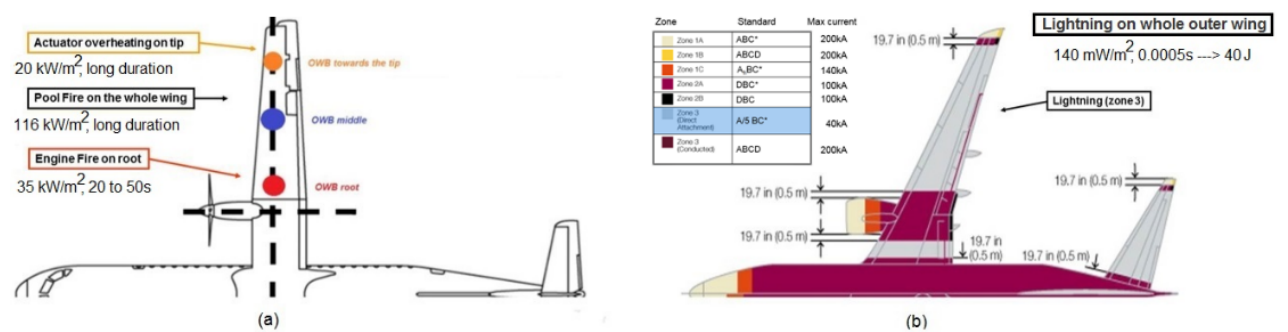

Fig. 8. Thermal threats and wing regions concerned.

Thermal conductivity tests have been performed by IMAST according to ASTM E1350 [6], in the range of temperature $30-300{ }^{\circ} \mathrm{C}$. The results on three different samples, two AFP composites representative of root and tip zones of the wing and a CS1 (HLU) composite, are 
reported in Figure 9(a). A comparison between IMAST thermal conductivity experimental results and SAMTECH values obtained from literature review [7] is also shown. The variation of thermal conductivity with temperature is resulted not negligible; the conductivity through the thickness of the composite laminate strongly depends, other than on resin content and fibre volume, also on the specimen layup.

Short beam shear tests in temperature have been executed by IMAST in a temperature controlled chamber according to ASTM D2344 [8]. Five HLU coupons, representative of the middle OWB panel have been loaded in a three-point bending mode at a rate of crosshead movement of $1 \mathrm{~mm} / \mathrm{min}$, for the temperatures: RT, 80, 130, 180 and $230{ }^{\circ} \mathrm{C}$. The short beam strength decreases with increasing temperature and Figures 9(b) and 9(c) show the results at $80^{\circ} \mathrm{C}$ and $230{ }^{\circ} \mathrm{C}$. The failure mode is resulted not correspondent to a sudden shear failure, as usual for this test, but it is more a continuous deformation without shear failure, probably due to the resin softening that absorbs the loads.

The fire reaction and resistance tests have been done to characterise the three main stages of a fire for the composite material: piloted ignition, fire development, fully developed fire.

Cone calorimeter tests [8] and flammability tests (UL94 [9] and Oxygen index test [10]) have been performed by IMAST on HLU panels of CS1 project and AFP panels (Figure 10(a)). The results show a heat release rate (HHR) proportional to coupon thickness and only resin contributes; heating phase up to $230^{\circ} \mathrm{C}$ and heating/blistering in range $230-380^{\circ} \mathrm{C}$; flammability over $380^{\circ} \mathrm{C}$ and a very low risk of fire spreading for the materials assessed. Propane burner torch tests have been performed on different AFP coupons representative of the root and tip zones of the wing, Figure $10(\mathrm{~b})$. For a heat flux of $116 \mathrm{~kW} / \mathrm{m}^{2}$ the temperature on the not exposed coupon face reaches $T>200^{\circ} \mathrm{C}$ in about $50 \mathrm{~s}$, and no flame penetration through the specimen in $5 \mathrm{~min}$ of tests due to the network of carbon fibers.

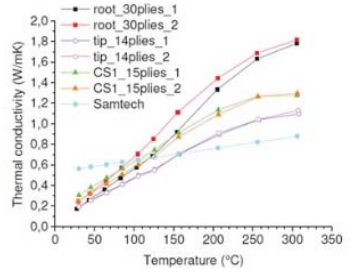

(a)

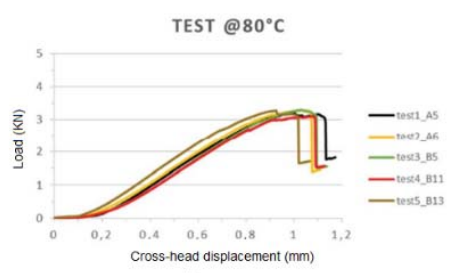

(b)

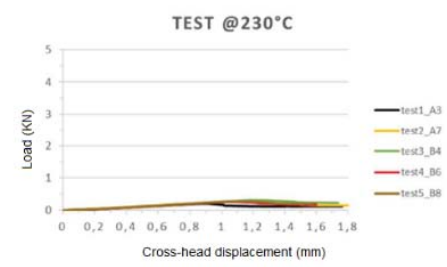

(c)

Fig. 9. Thermal conductivity values.

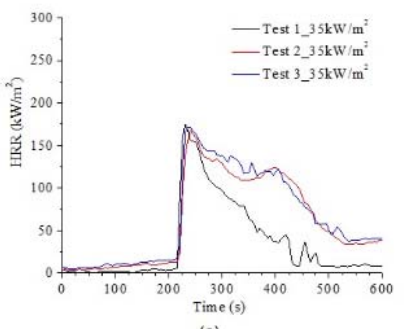

(a)

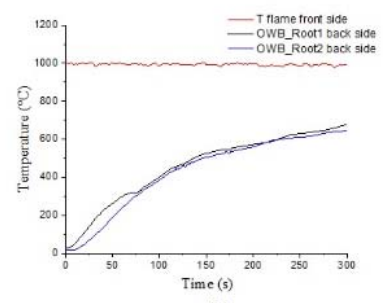

(b)

Fig. 10. Fire reaction test, fire resistance test.

The lightning tests performed by ONERA have assessed the composite materials behaviour under lightning. Measurements of coupons deformations, videos of arc root expansion and infrared measurements of temperature at the back of the coupons, have been performed. Four types of treatments have been applied: not protected, with paint, with metallic protection, with paint and metallic protection. A total of 13 tests have been performed, for AFP panels and for CS1 HLU panels, Figure 11. Some AFP panels were lightning protected with bronze 
mesh and some were painted with acrylic paint. The results have shown: non-protected and non-painted coupons exhibit severe damages and extended damage areas with delamination; the lightning protection prevents from most of the damage; the measurements made during the tests are in agreement with the damages observed after the tests.

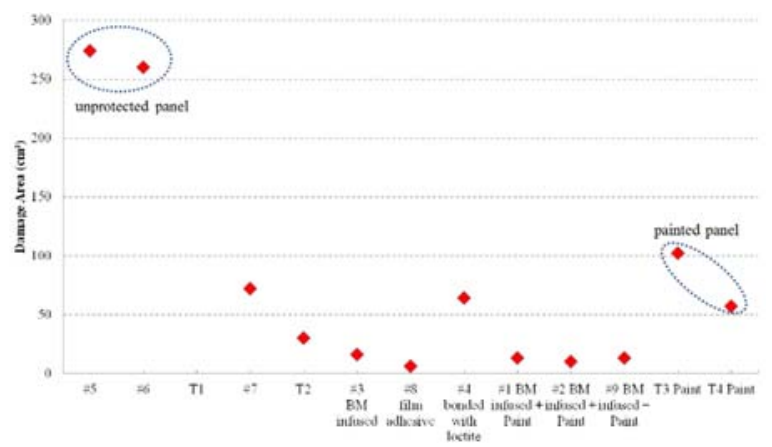

Fig. 11. Lightning tests.

IMAST and CIRA have performed different numerical activities to assess the behaviour of composite materials for the different thermal scenarios above identified. For these scenarios the results are in compliance with the specifications. As example, Figure 12 shows the results for the engine fire scenario.
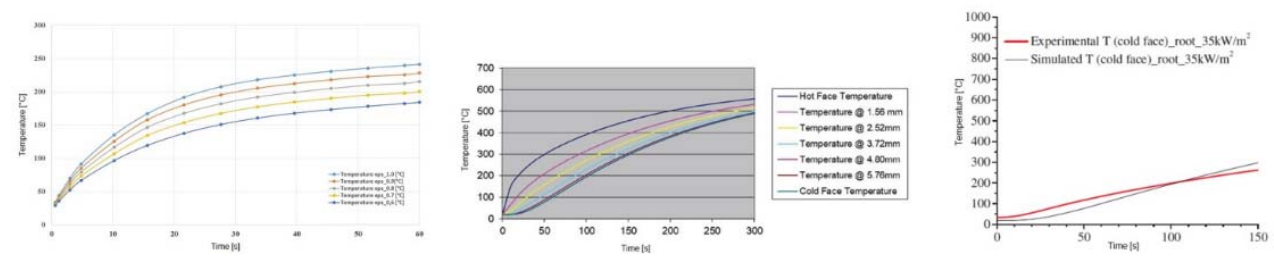

Fig. 12. Numerical analysis: engine fire scenario.

SAMTECH, by using Samcef software [11], has developed FE models for thermal simulations with degradation due to the temperature, and models used for thermo-mechanical simulations. As example, Figure 13 shows the results of the simulation of the Short Beam Shear Test (thermo-mechanical model). A good correlation was observed between Samcef results and experimental ones, under or over the matrix glass temperature, for the slopes, the maximum loads and the delamination locations, Figure 13(a). At mechanical glass temperature, the behaviour over maximum load as well as the fact that a permanent deformation occurs were correctly simulated, Figure 13(b).
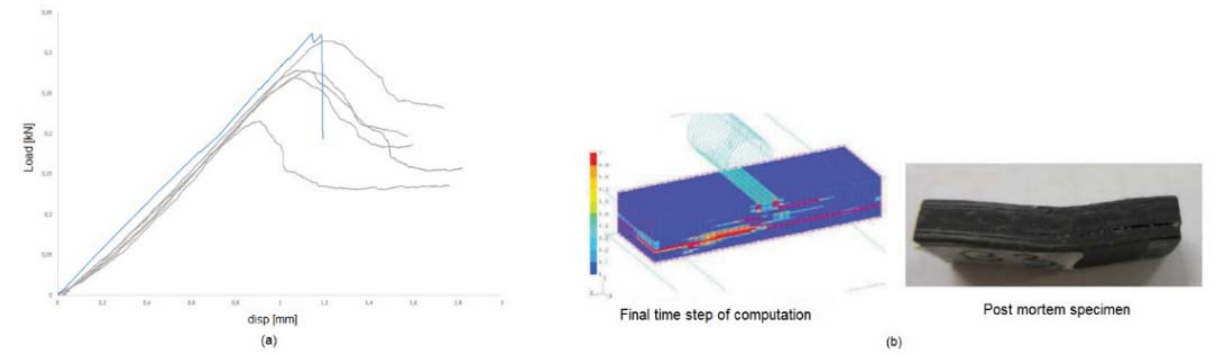

Fig. 13. Short Beam Shear Test numerical analysis (at $130{ }^{\circ} \mathrm{C}$ ): numerical and experimental load displacement curves; numerical and experimental delaminated zones at $130^{\circ} \mathrm{C}$ 


\section{Conclusions}

The results illustrated in this work are essential for the next steps of AirGreen 2 project. In particular, many useful information are emerged for the manufacturing of the next large curved stiffened panels with HLU and AFP techniques in terms of equipment set up and fine tuning, resin temperature tight control and full scale panel production test. Among the many outcomes: avoid omega tube crumple up and/or use of spiral for inlet/outlet vents, use of double bag, inlet tubes heated by resistance, temperature mapping along resin path during infusion phase, etc. The properties in operative conditions of the selected materials are resulted in agreement with the required specifications. Mechanical characterisation of HLU and AFP coupons are also in progress to compare the material allowables and the mechanical performance for the final design of the wing box.

\section{Acknowledgments}

The research leading to these results has received funding from Clean Sky 2 Joint Undertaking under the European Union's Horizon H2020 research and innovation programme, under grant agreement n. CS2-REG-GAM-2014-2015-01.

The Authors thank the colleagues that have worked and contributed to the activities shown in this work: V. Iannone (NOVOTECH), R. Zazza, B. Auriemma and S. D'Onofrio (SICAMB), M. Ferraiuolo, R. Scigliano and U. Mercurio (CIRA), S. Inserra (MAST), L. Chemartin, G. Leplat and C. Zaepffel (ONERA), A.C. Goupil (SAMTECH), V. Ascione (LEONARDO Aircraft).

\section{References}

1. F. Romano, R. Zazza, et al., Greener Aviation Conference Proceedings, Brussels, Belgium, Liquid Resin Infusion Technology For The Manufacturing Of Outer Wing Box Stiffened Panels (2016).

2. https://www.compositesworld.com/articles/resin-infused-ms-21-wings-and-wingbox

3. https:/www.esi-group.com/it/soluzioni-software/virtual-manufacturing/compositi

4. https://www.coriolis-composites.com/products/laser-heating-device.html

5. P. Wang, J. Molimard et al., ICCM 17 Conference Proceedings, Edinburgh UK, Monitoring the Liquid Resin Infusion (LRI) manufacturing process under industrial environment using distributed sensors, (2009).

6. ASTM E1350-97, Standard Test Methods for Testing Sheathed Thermocouples Prior to, During and After Installation, ASTM International, West Conshohocken, PA (1997)

7. V. Biasi, PhD Thesis, University of Toulouse and ISAE, Modélisation thermique de la dégradation d'un matériau composite soumis au feu, (2014).

8. ISO 5660-1, Reaction to Fire Tests, Heat release, smoke production and mass loss rate

9. ASTM D3801-10, Standard Test Method for Measuring the Comparative Burning Characteristics of Solid Plastics in a Vertical Position, ASTM International, West Conshohocken, PA (2010)

10. ASTM D2863, Standard Test Method for Measuring the Minimum Oxygen Concentration to Support Candle-Like Combustion of Plastics (Oxygen Index), ASTM International, West Conshohocken, PA, (2017)

11. https://www.plm.automation.siemens.com/fr/products/lms/samtech/index.shtml 